\title{
Article \\ Energy and Entropy Production of Nanofluid within an Annulus Partly Saturated by a Porous Region
}

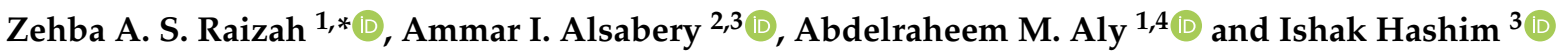 \\ 1 Department of Mathematics, College of Science, King Khalid University, Abha 62529, Saudi Arabia; \\ ababdallah@kku.edu.sa \\ 2 Refrigeration Air-Conditioning Technical Engineering Department, College of Technical Engineering, \\ The Islamic University, Najaf 54001, Iraq; ammar_e_2011@yahoo.com \\ 3 School of Mathematical Sciences, Faculty of Science \& Technology, University Kebangsaan Malaysia, \\ Bangi 43600, Malaysia; ishak_h@ukm.edu.my \\ 4 Department of Mathematics, Faculty of Science, South Valley University, Qena 83523, Egypt \\ * Correspondence: zaalrazh@kku.edu.sa
}

Citation: Raizah, Z.A.S.; Alsabery,

A.I.; Aly, A.M.; Hashim, I. Energy and Entropy Production of Nanofluid within an Annulus Partly Saturated by a Porous Region. Entropy 2021, 23, 1237. https://doi.org/10.3390/ e23101237

Academic Editor: Abderrahmane Baïri

Received: 3 August 2021

Accepted: 16 September 2021

Published: 22 September 2021

Publisher's Note: MDPI stays neutral with regard to jurisdictional claims in published maps and institutional affiliations.

Copyright: (c) 2021 by the authors. Licensee MDPI, Basel, Switzerland. This article is an open access article distributed under the terms and conditions of the Creative Commons Attribution (CC BY) license (https:// creativecommons.org/licenses/by/ $4.0 /)$.

\begin{abstract}
The flow and heat transfer fields from a nanofluid within a horizontal annulus partly saturated with a porous region are examined by the Galerkin weighted residual finite element technique scheme. The inner and the outer circular boundaries have hot and cold temperatures, respectively. Impacts of the wide ranges of the Darcy number, porosity, dimensionless length of the porous layer, and nanoparticle volume fractions on the streamlines, isotherms, and isentropic distributions are investigated. The primary outcomes revealed that the stream function value is powered by increasing the Darcy parameter and porosity and reduced by growing the porous region's area. The Bejan number and the average temperature are reduced by the increase in $D a$, porosity $\varepsilon$, and nanoparticles volume fractions $\phi$. The heat transfer through the nanofluid-porous layer was determined to be the best toward high rates of Darcy number, porosity, and volume fraction of nanofluid. Further, the local velocity and local temperature in the interface surface between nanofluid-porous layers obtain high values at the smallest area from the porous region $(D=0.4)$, and in contrast, the local heat transfer takes the lower value.
\end{abstract}

Keywords: natural convection; horizontal annulus; nanofluid-porous cavity; Darcy-Forchheimer model; entropy production

\section{Introduction}

Natural or free convection has vast, important applications in the industry field, such as electronic systems cooling [1], energy collectors solar [2], heat exchangers [3], electric ovens [4], desalination solar systems [5], melting [6,7] and freezing processes [8,9], etc. The heat transfer rate in natural convection is low as a result of the fluid velocity connected with natural convection is minimum. In natural convection, the fluid velocity is relatively low due to the fluid movement caused by the buoyancy force. Therefore, the heat transfer becomes weak also. For these reasons, a technique must be found to enhance the natural convection of heat transfer inside enclosures. One of the methods is to add nanoparticles to the base fluids, or use the porous media inside the enclosures.

The porous medium, for example, is a hard sedimentary rock, wood, bread, and a lung or a substance consisting of a solid structure with an interconnected vacuum. The fluid flow through the interconnected void depends on the porosity and the permeability of the media. Regarding shape and size, when the distribution of pores is unequal, the media is called natural porous media, for example, sandstone and bones [10]. Over the years, numerous researchers have made great progress in the subject of porous media inside enclosures. Porous media are perfect for improving conduction inside enclosures. On the other hand, it causes resistance to the fluid flow and weakens convection. Nanomaterials 
of infinitesimal sizes play a successful role in the heat transfer, so the nanofluids are used simultaneously with porous media to improve heat transfer [11-13]. Toosi and Siavashi [11] investigated the natural convection inside a square cavity partially filled with porous media and of $\mathrm{Cu}$-water nanofluid. They used Corcione's model for a two-phase model and Darcy-Brinkman-Forchheimer to simulate fluid flow in porous media. They found that the convection becomes weak by using nanofluid and porous media, and also the conduction can be strengthened. Baghsaz et al. performed a study on natural convection heat transfer and entropy generation inside a porous cavity filled with $\mathrm{Al}_{2} \mathrm{O}_{3}$ /water nanofluid. They found that the natural convection heat transfer, circulation, and irreversibility of the production increased clearly by increasing the Rayleigh number $(R a)$ and Darcy number $(D a)$. Miroshnichenko et al. [14] simulated the natural convection in an open cavity containing porous layers and filled with alumina/water nanofluid, which penetrated the cavity from the open boundary. They used a single-phase nanofluid approach and the Brinkman-extended Darcy model for porous layers. Their results showed that the heat transfer rate was increased by the expansion of the range between the wall and the porous layer. Cho [15] analyzed the heat transfer of natural convection inside a porous cavity filled with nanofluid and containing a partially-heated vertical wall. He showed that for high values of $D a$ and $R a$, convection heat transfer dominates. Thus, the entropy generation and mean Nusselt number increases, whereas the Bejan number decreases. Using the finite volume method, Selimefendigil and Öztop [16] performed a study on magnetohydrodynamics forced convection of carbon nanotube of water nanofluid in a U-shaped cavity containing a porous region under the impact of a wall rippling. They illustrated that the variation of the Reynolds number and permeability of the porous medium affected the flow field and heat transfer. Using a Local Thermal Non-Equilibrium (LTNE) approach, Mehryan et al. [17] reported the fluid flow and heat transfer of natural convection inside a porous enclosure filled with Ag-MgO/water nanofluids. Alsabery et al. [18], Raizah et al. [19], Aly and Raizah [20], Javaherdeh et al. [21], Rao and Barman [22], Wang et al. [23] and Esfe et al. [24] executed numerical imitation studies to analyze the natural convection heat transfer of a nanofluid in porous cavities. They illustrated that one could enhance the heat transfer performance by assuming both porous media and nanofluid.

As noticed from the earlier research surveys, and to the best of the authors' knowledge, there is no outcome with the convection flow within horizontal annulus filled with nanofluid superposed porous layers. Accordingly, this work introduces the understanding of nanofluid superposed horizontal annulus porous layers via the fluid flow and heat transfer distributions.

\section{Mathematical Formulation}

The steady natural convection and heat transfer of water- $\mathrm{Al}_{2} \mathrm{O}_{3}$ nanofluid within an annulus by radius $r r$ and having an inner hot $\left(T_{h}\right)$ cylinder by radius $r$ is explained in Figure 1. The analyzed composite annulus cavity is divided into two segments, the first one (outer portion) is filled by nanofluid and the second one (inner portion) is loaded by a porous region saturated by a nanofluid. The outer surface is fixed with a cold temperature of $T_{\mathcal{C}}$. The edges of the domain (except for the interface surface between the nanofluid-porous layers) are supposed to remain impermeable. The mixed liquid inside the composite cavity performs as a water-based nanofluid holding $\mathrm{Al}_{2} \mathrm{O}_{3}$ nanoparticles. The Forchheimer-Brinkman-extended Darcy approach and the Boussinesq approximation remain appropriate. In contrast, the nanofluid phase's convection and the solid matrix are in the local thermodynamic equilibrium condition. The set of porous media applied in the following output is glass balls $\left(k_{m}=1.05 \mathrm{~W} / \mathrm{m} \cdot{ }^{\circ} \mathrm{C}\right)$. Examining earlier specified hypotheses, the continuity, momentum and energy equations concerning the Newtonian laminar flow survive, formulated as:

For the nanofluid region: 


$$
\begin{aligned}
& \frac{\partial u_{n f}}{\partial x}+\frac{\partial v_{n f}}{\partial y}=0 \\
& u_{n f} \frac{\partial u_{n f}}{\partial x}+v_{n f} \frac{\partial u_{n f}}{\partial y}=-\frac{1}{\rho_{n f}} \frac{\partial p}{\partial x}+\frac{\mu_{n f}}{\rho_{n f}}\left(\frac{\partial^{2} u_{n f}}{\partial x^{2}}+\frac{\partial^{2} u_{n f}}{\partial y^{2}}\right) \\
& u_{n f} \frac{\partial v_{n f}}{\partial x}+v_{n f} \frac{\partial v_{n f}}{\partial y}=-\frac{1}{\rho_{n f}} \frac{\partial p}{\partial y}+\frac{\mu_{n f}}{\rho_{n f}}\left(\frac{\partial^{2} v_{n f}}{\partial x^{2}}+\frac{\partial^{2} v_{n f}}{\partial y^{2}}\right)+\beta_{n f} g\left(T_{h}-T_{c}\right) \\
& u_{n f} \frac{\partial T_{n f}}{\partial x}+v_{n f} \frac{\partial T_{n f}}{\partial y}=\frac{k_{n f}}{\left(\rho C_{p}\right)_{n f}}\left(\frac{\partial^{2} T_{n f}}{\partial x^{2}}+\frac{\partial^{2} T_{n f}}{\partial y^{2}}\right) .
\end{aligned}
$$

For the porous region:

$$
\begin{aligned}
& \frac{\partial u_{m}}{\partial x}+\frac{\partial v_{m}}{\partial y}=0, \\
& \frac{\rho_{n f}}{\varepsilon^{2}}\left(u_{m} \frac{\partial u_{m}}{\partial x}+v_{m} \frac{\partial u_{m}}{\partial y}\right)=-\frac{\partial p}{\partial x}+\frac{\mu_{n f}}{\varepsilon}\left(\frac{\partial^{2} u_{m}}{\partial x^{2}}+\frac{\partial^{2} u_{m}}{\partial y^{2}}\right) \\
& \quad-\left(\frac{\mu_{n f}}{K} u_{m}-\frac{1.75}{\sqrt{150} \varepsilon^{3 / 2}} \frac{\rho_{n f} u_{m}|\mathbf{u}|}{\sqrt{K}}\right), \\
& \frac{\rho_{n f}}{\varepsilon^{2}}\left(u_{m} \frac{\partial v_{m}}{\partial x}+v_{m} \frac{\partial v_{m}}{\partial y}\right)=-\frac{\partial p}{\partial y}+\frac{\mu_{n f}}{\varepsilon}\left(\frac{\partial^{2} v_{m}}{\partial x^{2}}+\frac{\partial^{2} v_{m}}{\partial y^{2}}\right) \\
& \quad-\left(\frac{\mu_{n f}}{K} v_{m}-\frac{1.75}{\sqrt{150} \varepsilon^{3 / 2}} \frac{\rho_{n f} v_{m}|\mathbf{u}|}{\sqrt{K}}\right)+(\rho \beta)_{n f} \mathrm{~g}\left(T_{h}-T_{c}\right), \\
& u_{m} \frac{\partial T_{m}}{\partial x}+v_{m} \frac{\partial T_{m}}{\partial y}=\frac{\varepsilon k_{n f}}{\left(\rho C_{p}\right)_{n f}}\left(\frac{\partial^{2} T_{m}}{\partial x^{2}}+\frac{\partial^{2} T_{m}}{\partial y^{2}}\right) .
\end{aligned}
$$

The subscripts $n f$ and $m$ view the nanofluid layer and porous layer. $x$ and $y$ are the fluid velocity elements, $|\mathbf{u}|=\sqrt{u^{2}+v^{2}}$ denotes the Darcy velocity, $g$ displays the acceleration due to gravity, $\varepsilon$ signifies the porosity of the medium and $K$ is the permeability of the porous medium, which is determined as [25]:

$$
K=\frac{\varepsilon^{3} d_{m}^{2}}{150(1-\varepsilon)^{2}}
$$

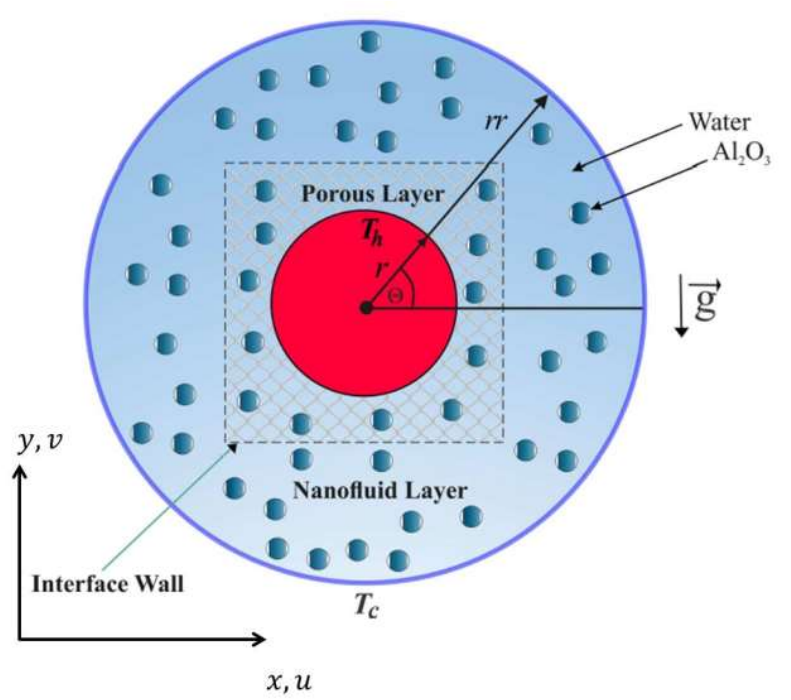

Figure 1. Schematic representation concerning the convection flow in annulus composite. 
Here, $d_{m}$ represents the average particle size of the porous region.

The thermo-physical characteristics regarding the adopted nanofluid for $33 \mathrm{~nm}$ particle-size will be prepared as [26]:

$$
\begin{aligned}
& \left(\rho C_{p}\right)_{n f}=(1-\phi)\left(\rho C_{p}\right)_{f}+\phi\left(\rho C_{p}\right)_{p} \\
& \rho_{n f}=(1-\phi) \rho_{f}+\phi \rho_{p} \\
& (\rho \beta)_{n f}=(1-\phi)(\rho \beta)_{f}+\phi(\rho \beta)_{p} \\
& \frac{\mu_{n f}}{\mu_{f}}=\frac{1}{1-34.87\left(\frac{d_{p}}{d_{f}}\right)^{-0.3} \phi^{1.03}}, \\
& \frac{k_{n f}}{k_{f}}=1+4.4 \operatorname{Re}_{B}^{0.4} \operatorname{Pr}^{0.66}\left(\frac{T}{T_{f r}}\right)^{10}\left(\frac{k_{p}}{k_{f}}\right)^{0.03} \phi^{0.66} .
\end{aligned}
$$

where $\operatorname{Re}_{B}$ is defined as

$$
\operatorname{Re}_{B}=\frac{\rho_{f} u_{B} d_{p}}{\mu_{f}}, \quad u_{B}=\frac{2 k_{b} T}{\pi \mu_{f} d_{p}^{2}} .
$$

The molecular diameter of water $\left(d_{f}\right)$ is given as [26]

$$
d_{f}=0.1\left[\frac{6 M}{N \pi \rho_{f}}\right]^{\frac{1}{3}}
$$

Presently we present the employed non-dimensional variables:

$$
\begin{aligned}
& (X, Y)=\frac{(x, y)}{L}, U_{n f, m}=\frac{u_{n f, m} L}{\alpha_{f}}, V_{n f, m}=\frac{v_{n f, m} L}{\alpha_{f}}, \theta_{n f}=\frac{T_{n f}-T_{c}}{T_{h}-T_{c}} \\
& \theta_{m}=\frac{T_{m}-T_{c}}{T_{h}-T_{c}}, P=\frac{p L^{2}}{\rho_{f} \alpha_{f}^{2}}, k_{e f f}=\varepsilon k_{n f}+(1-\varepsilon) k_{m}, C_{F}=\frac{1.75}{\sqrt{150}} .
\end{aligned}
$$

The set scheme leads to the following dimensionless governing equations:

In the nanofluid region:

$$
\begin{aligned}
& \frac{\partial U_{n f}}{\partial X}+\frac{\partial V_{n f}}{\partial Y}=0, \\
& U_{n f} \frac{\partial U_{n f}}{\partial X}+V_{n f} \frac{\partial U_{n f}}{\partial Y}=-\frac{\partial P}{\partial X}+\operatorname{Pr} \frac{\rho_{f}}{\rho_{n f}} \frac{\mu_{n f}}{\mu_{f}}\left(\frac{\partial^{2} U_{n f}}{\partial x^{2}}+\frac{\partial^{2} U_{n f}}{\partial Y^{2}}\right), \\
& U_{n f} \frac{\partial V_{n f}}{\partial X}+V_{n f} \frac{\partial V_{n f}}{\partial Y}=-\frac{\partial P}{\partial Y}+\operatorname{Pr} \frac{\rho_{f}}{\rho_{n f}} \frac{\mu_{n f}}{\mu_{f}}\left(\frac{\partial^{2} V_{n f}}{\partial X^{2}}+\frac{\partial^{2} V_{n f}}{\partial Y^{2}}\right) \\
& \quad+\frac{(\rho \beta)_{n f}}{\rho_{n f} \beta_{f}} \operatorname{RaPr} \theta_{n f}, \\
& U_{n f} \frac{\partial \theta_{n f}}{\partial X}+V_{n f} \frac{\partial \theta_{n f}}{\partial Y}=\frac{\left(\rho C_{p}\right)_{f}}{\left(\rho C_{p}\right)_{n f}} \frac{k_{n f}}{k_{f}}\left(\frac{\partial^{2} \theta_{n f}}{\partial X^{2}}+\frac{\partial^{2} \theta_{n f}}{\partial Y^{2}}\right) .
\end{aligned}
$$

In the porous region:

$$
\frac{\partial U_{m}}{\partial X}+\frac{\partial V_{m}}{\partial Y}=0
$$




$$
\begin{gathered}
\frac{1}{\varepsilon^{2}}\left(U_{m} \frac{\partial U_{m}}{\partial X}+V_{m} \frac{\partial U_{m}}{\partial Y}\right)=-\frac{\partial P}{\partial X}+\frac{\rho_{f}}{\rho_{n f}} \frac{\mu_{n f}}{\mu_{f}} \frac{\operatorname{Pr}}{\varepsilon}\left(\frac{\partial^{2} U_{m}}{\partial X^{2}}+\frac{\partial^{2} U_{m}}{\partial Y^{2}}\right) \\
-\frac{\rho_{f}}{\rho_{n f}} \frac{\mu_{n f}}{\mu_{f}} \frac{\operatorname{Pr}}{D a} U_{m}-\frac{C_{F} \sqrt{U_{m}^{2}+V_{m}^{2}}}{\sqrt{D a}} \frac{U_{m}}{\varepsilon^{3 / 2}} \\
\frac{1}{\varepsilon^{2}}\left(U_{m} \frac{\partial V_{m}}{\partial X}+V_{m} \frac{\partial V_{m}}{\partial Y}\right)=-\frac{\partial P}{\partial Y}+\frac{\rho_{f}}{\rho_{n f}} \frac{\mu_{n f}}{\mu_{f}} \frac{\operatorname{Pr}}{\varepsilon}\left(\frac{\partial^{2} V_{m}}{\partial X^{2}}+\frac{\partial^{2} V_{m}}{\partial Y^{2}}\right) \\
-\frac{\rho_{f}}{\rho_{n f}} \frac{\mu_{n f}}{\mu_{f}} \frac{\operatorname{Pr}}{D a} V_{m}-\frac{C_{F} \sqrt{U_{m}^{2}+V_{m}^{2}}}{\sqrt{D a}} \frac{V_{m}}{\varepsilon^{3 / 2}}+\frac{(\rho \beta)_{n f}}{\rho_{n f} \beta_{f}} R a \operatorname{Pr} \theta_{m}, \\
\frac{1}{\varepsilon}\left(U_{m} \frac{\partial \theta_{m}}{\partial X}+V_{m} \frac{\partial \theta_{m}}{\partial Y}\right)=\frac{k_{e f f}}{k_{f}} \frac{\left(\rho C_{p}\right)_{f}}{\left(\rho C_{p}\right)_{n f}}\left(\frac{\partial^{2} \theta_{m}}{\partial X^{2}}+\frac{\partial^{2} \theta_{m}}{\partial Y^{2}}\right) .
\end{gathered}
$$

The dimensionless boundary conditions of Equations (18)-(25) are:

$$
\begin{aligned}
& \text { In the boundary of inner cylinder: } U=V=0, \theta_{m}=1, \\
& \text { In the boundary of outer cylinder: } U=V=0, \theta_{n f}=0,
\end{aligned}
$$

and the dimensionless boundary forms toward the interface between the nanofluid and porous layers will be obtained from (1) the continuity of tangential and normal velocities, (2) shear and normal stresses, (3) temperature and the heat flux that crossed the central interface and allowing an identical dynamic viscosity $\left(\mu_{n f}=\mu_{m}\right)$ into both layers. Therefore, the interface dimensionless boundary conditions can be addressed as the following:

$$
\begin{aligned}
\left.\theta_{n f}\right|_{Y=D^{+}} & =\left.\theta_{m}\right|_{Y=D^{-}}, \\
\left.\frac{\partial \theta_{n f}}{\partial Y}\right|_{Y=D^{+}} & =\left.\frac{k_{e f f}}{k_{n f}} \frac{\partial \theta_{m}}{\partial Y}\right|_{Y=D^{-}}, \\
\left.U_{n f}\right|_{Y=D^{+}} & =\left.U_{m}\right|_{Y=D^{-}}, \\
\left.V_{n f}\right|_{Y=D^{+}} & =\left.V_{m}\right|_{Y=D^{-}},
\end{aligned}
$$

here $D$ denotes the porous layer's thickness, and the subscripts + and - indicated that the corresponding measures are estimated while addressing the interface of the nanofluid and porous layers, respectively. $R a=\frac{g \beta_{f}\left(T_{h}-T_{c}\right) L^{3}}{v_{f} \alpha_{f}}$ and $\operatorname{Pr}=\frac{v_{f}}{\alpha_{f}}$ signify the Rayleigh number and Prandtl number related to the used base liquid.

Local Nusselt numbers $\left(N u_{n f}\right.$ and $\left.N u_{i}\right)$ at the inner cylinder and interface wall are, respectively, as follows:

$$
\begin{gathered}
N u_{n f}=\frac{k_{e f f}}{k_{f}}\left(\frac{\partial \theta}{\partial n}\right)_{n} . \\
N u_{i}=\frac{k_{n f}}{k_{f}}\left(\frac{\partial \theta}{\partial D}\right)_{D},
\end{gathered}
$$

here $n$ and $D$ determine the entire length of the inner cylinder heat source and interface wall, respectively.

Lastly, the average Nusselt number at the inner heated cylinder is addressed by the following:

$$
\overline{N u}_{n f}=\int_{0}^{n} N u_{n f} \mathrm{~d} n,
$$

The entropy production related to the nanofluid layer is provided by [27-29]:

$$
S_{n f}=\frac{k_{n f}}{T_{0}^{2}}\left[\left(\frac{\partial T}{\partial x}\right)^{2}+\left(\frac{\partial T}{\partial y}\right)^{2}\right]+\frac{\mu_{n f}}{T_{0}}\left[2\left(\frac{\partial u}{\partial x}\right)^{2}+2\left(\frac{\partial v}{\partial y}\right)^{2}+\left(\frac{\partial u}{\partial x}+\frac{\partial v}{\partial x}\right)^{2}\right] .
$$


While the entropy production-related to the porous layer is addressed by [30]:

$$
\begin{aligned}
S_{m}= & \frac{k_{e f f}}{T_{0}^{2}}\left[\left(\frac{\partial T}{\partial x}\right)^{2}+\left(\frac{\partial T}{\partial y}\right)^{2}\right]+\frac{\mu_{f}}{T_{0}}\left[2\left(\frac{\partial u}{\partial x}\right)^{2}+2\left(\frac{\partial v}{\partial y}\right)^{2}+\left(\frac{\partial u}{\partial x}+\frac{\partial v}{\partial x}\right)^{2}\right] \\
& +\frac{\mu_{f}}{K T_{0}}\left(u^{2}+v^{2}\right) .
\end{aligned}
$$

In the dimensionless pattern, Equation (35) can be signified as:

$$
\begin{aligned}
& S_{\mathrm{GEN}, n f}=\frac{k_{n f}}{k_{f}}\left[\left(\frac{\partial \theta}{\partial X}\right)^{2}+\left(\frac{\partial \theta}{\partial Y}\right)^{2}\right] \\
& +\frac{\mu_{n f}}{\mu_{f}} N_{n f}\left\{2\left[\left(\frac{\partial U}{\partial X}\right)^{2}+\left(\frac{\partial V}{\partial Y}\right)^{2}\right]+\left(\frac{\partial^{2} U}{\partial Y^{2}}+\frac{\partial^{2} V}{\partial X^{2}}\right)^{2}\right\},
\end{aligned}
$$

where $N_{n f}$ denotes the irreversibility distribution ratio within the nanofluid region and can be represented by:

$$
N_{n f}=\frac{\mu_{f} T_{0}}{k_{f}}\left(\frac{\alpha_{f}}{L(\Delta T)}\right)^{2}
$$

and $S_{\mathrm{GEN}, n f}$ explains the dimensionless entropy production rate:

$$
S_{\mathrm{GEN}, n f}=S_{\mathrm{gen}, n f} \frac{T_{0}^{2} L^{2}}{k_{f}(\Delta T)^{2}} .
$$

The terms of Equation (39) will be divided based on the following scheme:

$$
S_{\mathrm{GEN}, n f}=S_{n f, \theta}+S_{n f, \Psi},
$$

here, $S_{n f, \theta}$ and $S_{n f, \Psi}$ obtain the entropy generation regarding the heat transfer irreversibility (HTI) and fluid friction irreversibility (FFI) of the nanofluid layer, respectively.

$$
\begin{aligned}
& S_{n f, \theta}=\frac{k_{n f}}{k_{f}}\left[\left(\frac{\partial \theta}{\partial X}\right)^{2}+\left(\frac{\partial \theta}{\partial Y}\right)^{2}\right], \\
& S_{n f, \Psi}=\frac{\mu_{n f}}{\mu_{f}} N_{n f}\left\{2\left[\left(\frac{\partial U}{\partial X}\right)^{2}+\left(\frac{\partial V}{\partial Y}\right)^{2}\right]+\left(\frac{\partial^{2} U}{\partial Y^{2}}+\frac{\partial^{2} V}{\partial X^{2}}\right)^{2}\right\} .
\end{aligned}
$$

Into the dimensionless structure, Equation (36) remains exposed as:

$$
\begin{gathered}
S_{\mathrm{GEN}, m}=\frac{k_{e f f}}{k_{f}}\left[\left(\frac{\partial \theta}{\partial X}\right)^{2}+\left(\frac{\partial \theta}{\partial Y}\right)^{2}\right]+\frac{\mu_{n f}}{\mu_{f}} N_{m} \times \\
\left\{D a\left[2\left(\left(\frac{\partial U}{\partial X}\right)^{2}+\left(\frac{\partial V}{\partial Y}\right)^{2}\right)+\left(\frac{\partial^{2} U}{\partial Y^{2}}+\frac{\partial^{2} V}{\partial X^{2}}\right)^{2}\right]+\left(U^{2}+V^{2}\right)\right\},
\end{gathered}
$$

where $N_{m}=\frac{\mu_{f} T_{0}}{k_{f}}\left(\frac{\alpha_{f}^{2}}{K(\Delta T)^{2}}\right)$ is the irreversibility distribution ratio in the porous layer and $S_{\mathrm{GEN}, m}=S_{\text {gen }, m} \frac{T_{0}^{2} L^{2}}{k_{f}(\Delta T)^{2}}$.

The terms of Equation (43) can be separated into the following form:

$$
S_{\mathrm{GEN}, m}=S_{m, \theta}+S_{m, \Psi},
$$


where $S_{m, \theta}$ and $S_{m, \Psi}$ are the entropy generation due to heat transfer irreversibility (HTI) and fluid friction irreversibility (FFI) of the porous layer, respectively.

$$
\begin{aligned}
& S_{m, \theta}=\frac{k_{e f f}}{k_{f}}\left[\left(\frac{\partial \theta}{\partial X}\right)^{2}+\left(\frac{\partial \theta}{\partial Y}\right)^{2}\right], \\
& S_{m, \Psi}=\frac{\mu_{n f}}{\mu_{f}} N_{m} \times \\
& \quad\left\{D a\left[2\left(\left(\frac{\partial U}{\partial X}\right)^{2}+\left(\frac{\partial V}{\partial Y}\right)^{2}\right)+\left(\frac{\partial^{2} U}{\partial Y^{2}}+\frac{\partial^{2} V}{\partial X^{2}}\right)^{2}\right]+\left(U^{2}+V^{2}\right)\right\} .
\end{aligned}
$$

In the dimensionless form, the local entropy generation can be expressed as:

$$
S_{\mathrm{GEN}}=S_{\mathrm{GEN}, n f}+S_{\mathrm{GEN}, m}
$$

The global entropy generation (GEG) is obtained by integrating Equation (47) over the domain

$$
\mathrm{GEG}=\int S_{\mathrm{GEN}} \mathrm{dXd} Y=\int S_{\mathrm{GEN}, n f} \mathrm{dXd} Y+\int S_{\mathrm{GEN}, m} \mathrm{~d} X \mathrm{~d} Y .
$$

The Bejan number $B e$ is defined as:

$$
B e=\frac{\int S_{n f, \theta} \mathrm{d} X \mathrm{~d} Y+\int S_{m, \theta} \mathrm{d} X \mathrm{~d} Y}{\mathrm{GEG}} .
$$

When $B e>0.5$, the HTI is dominant, while when $B e<0.5$, the FFI is dominant.

\section{Numerical Method and Validation}

The governing dimensionless equations Equations (18)-(25) ruled with the boundary conditions Equations (26)-(31) are solved by the Galerkin weighted residual finite element technique. The computational region does discretize toward small triangular portions, as shown in Figure 2.

These small triangular Lagrange components with various forms are applied to each flow variable within the computational region. Residuals for each conservation equation exist and are accomplished through substituting the approximations within the governing equations. The Newton-Raphson iteration algorithm is adopted for clarifying the nonlinear expressions into the momentum equations. The convergence from the current numerical solution is considered, while the corresponding error toward each of the variables fills the resulting convergence criteria:

$$
\left|\frac{\Gamma^{i+1}-\Gamma^{i}}{\Gamma^{i+1}}\right| \leq 10^{-6}
$$

For confirming the independence regarding the existing numerical solution at the grid size of the numerical region, different grid sizes are used to determine the average Nusselt number $\left(\overline{N u}_{n f}\right)$, average temperature $\left(\theta_{\text {avg }}\right)$ and Bejan number $(B e)$ for the case of $R a=10^{6}, D a=10^{-3}, \phi=0.02, D=0.5$ and $\varepsilon=0.5$. The outcomes given in Table 1 designate irrelevant variations for the G5 grids and beyond. Hence, for all calculations into this work and similar obstacles to this subsection, the G5 uniform grid is applied. 


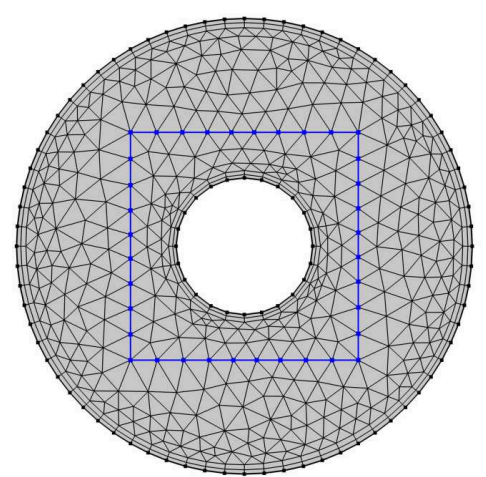

(a)

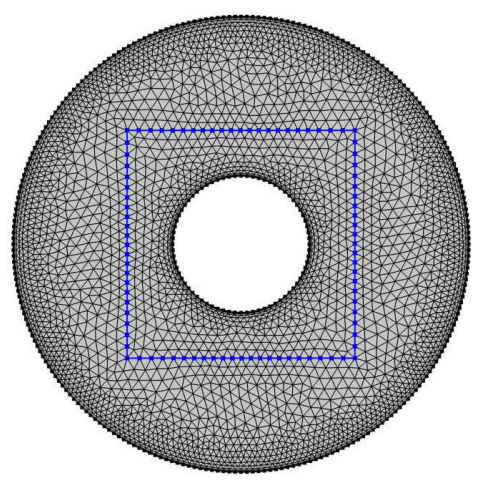

(b)

Figure 2. Framework configuration of the FEM for the grid dimension of (a) 958 and (b) 6026 components.

Table 1. Grid testing for $\overline{N u}_{n f}, \theta_{\text {avg }}$ and $B e$ at different grid sizes for $R a=10^{6}, D a=10^{-3}, \phi=0.02$, $\varepsilon=0.5$ and $D=0.5$.

\begin{tabular}{ccccc}
\hline Grid Size & Number of Elements & $\overline{\boldsymbol{N u}}_{\boldsymbol{n} f}$ & $\boldsymbol{\theta}_{\text {avg }}$ & $\boldsymbol{B} \boldsymbol{e}$ \\
\hline G1 & 486 & 19.519 & 0.30926 & 0.011058 \\
G2 & 958 & 19.525 & 0.29652 & 0.012067 \\
G3 & 1310 & 19.533 & 0.29534 & 0.016238 \\
G4 & 2008 & 19.539 & 0.29143 & 0.01726 \\
G5 & 6026 & 19.539 & 0.28799 & 0.018096 \\
G6 & 17324 & 19.543 & 0.28742 & 0.018244 \\
\hline
\end{tabular}

Regarding the validation for the numerical data, the current results have been compared with earlier experimental and numerical outcomes achieved by Kuehn and Goldstein [31], as shown in Figure 3 concerning the natural convection heat transfer case within a horizontal concentric annulus saturated with pure fluid (water). Furthermore, the results are considered among earlier declared experimental results obtained by Beckermann et al. [32] via a natural convection problem inside a square cavity having both fluid and porous layers, as implemented in Figure 4 . As explained by the earlier performed comparisons, the numerical outcomes of the present numerical code are essential to a high degree of reliability.

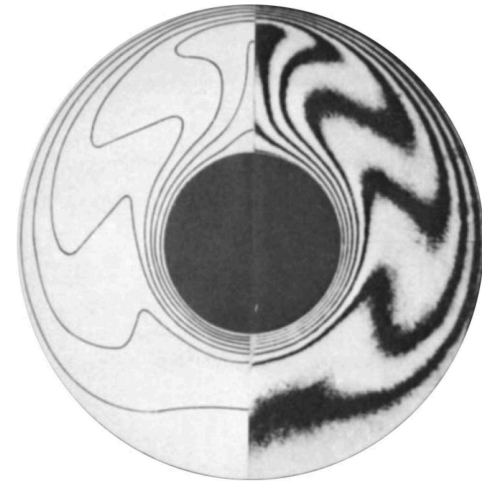

(a)

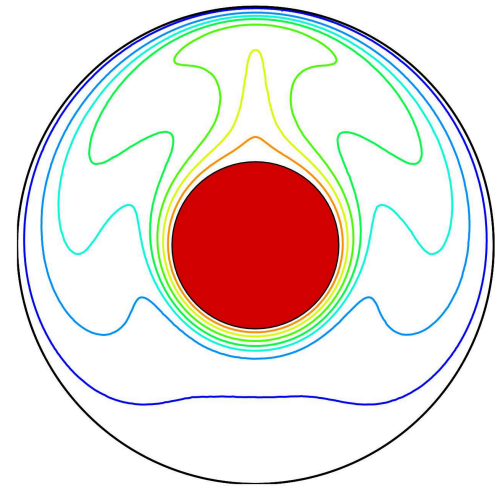

(b)

Figure 3. Isotherms of (a) Kuehn and Goldstein [31]; numerical result (left) and experimental result (right), and (b) present study for $R a=5 \times 10^{4}$ and $\operatorname{Pr}=0.7$. 

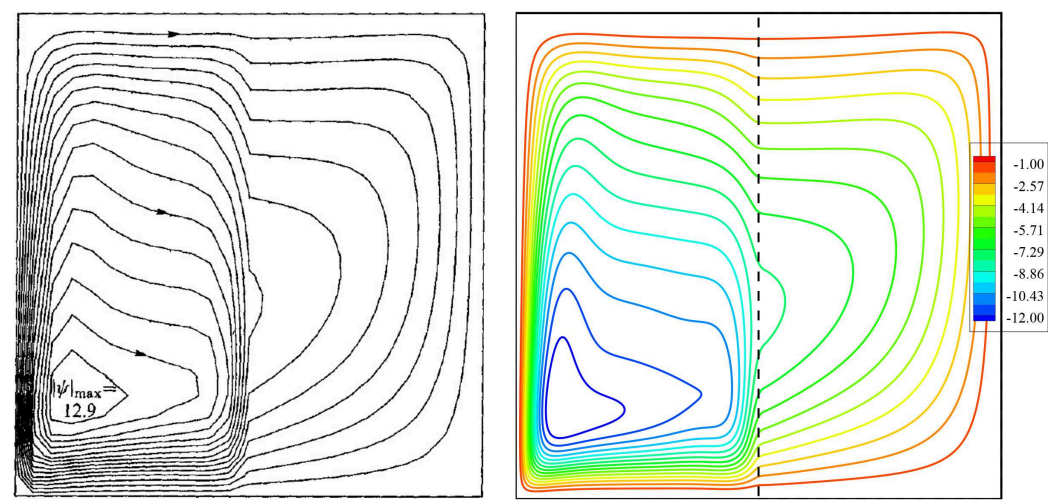

(a)
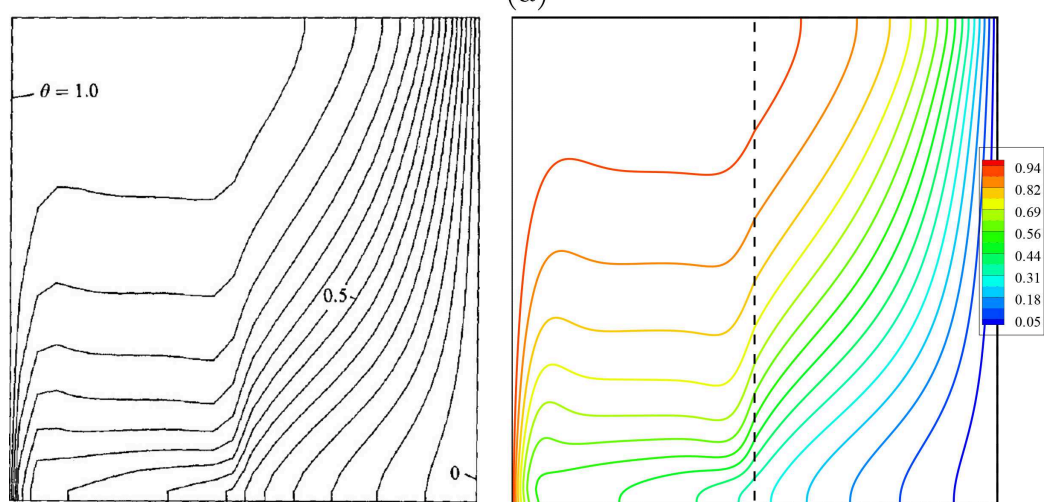

(b)

Figure 4. (a) Streamlines of Beckermann et al. [32] (left) and the wpresent study (right); (b) isotherms for $R a=3.70 \times 10^{6}, D a=1.370 \times 10^{-5}, \varepsilon=0.9, D=0.5 \mathrm{~N}=0, \frac{k_{e f f}}{k_{f}}=1.362$ and $\operatorname{Pr}=6.44$.

\section{Results and Discussion}

The outcomes described by streamlines, isotherms, and isentropic distributions are addressed within this segment. We have modified the following four parameters; Darcy number $\left(10^{-6} \leq D a \leq 10^{-2}\right)$, nanoparticle volume fraction $(0 \leq \phi \leq 0.04)$, dimensionless porous layer length $(0.4 \leq D \leq 0.7)$ and the porosity of the medium $(0.2 \leq \varepsilon \leq 0.8)$. The values of the Rayleigh number and Prandtl number are fixed at $R a=10^{6}$ and $\operatorname{Pr}=$ 4.623, respectively. Table 2 displays the thermophysical properties of the base fluid (water) and the solid $\mathrm{Al}_{2} \mathrm{O}_{3}$ phases at $T=310 \mathrm{~K}$.

Table 2. Thermophysical characteristics concerning pure liquid (water) and $\mathrm{Al}_{2} \mathrm{O}_{3}$ nanoparticles at $T=310 \mathrm{~K}[33]$.

\begin{tabular}{ccc}
\hline Physical Properties & Fluid Phase (Water) & $\mathbf{A l}_{\mathbf{2}} \mathbf{O}_{\mathbf{3}}$ \\
\hline$C_{p}(\mathrm{~J} / \mathrm{kgK})$ & 4178 & 765 \\
$\rho\left(\mathrm{kg} / \mathrm{m}^{3}\right)$ & 993 & 3970 \\
$k\left(\mathrm{Wm}^{-1} \mathrm{~K}^{-1}\right)$ & 0.628 & 40 \\
$\beta \times 10^{5}(1 / \mathrm{K})$ & 36.2 & 0.85 \\
$\mu \times 10^{6}(\mathrm{~kg} / \mathrm{ms})$ & 695 & - \\
$d_{p}(\mathrm{~nm})$ & 0.385 & 33 \\
\hline
\end{tabular}

The reliance of the streamlines, isotherms, and isentropic lines on the Darcy numbers (Da) when $\phi=0.02, D=0.5$ and $\varepsilon=0.5$ has been shown in Figure 5 . It is remarked that as $D a$ increases from $10^{-5}$ to $10^{-2}$, the absolute value of the streamline's maximum $|\psi|_{\max }$ is strongly increasing. The reason is the altitude porous resistance of the nanofluid flow at lower $D a$. Thus, the streamlines' intensity around a circular cylinder within an annulus raises as $D a$ increases. The isothermal lines filled the annulus at the lower Darcy parameter 
$D a=10^{-5}$ compared to the higher Darcy parameter $D a=10^{-2}$. The isotherms' strength is rising across the top area of an annulus according to the influence in $D a$. Thus, the intensity of the isentropic lines is strongly rising as $D a$ increases. At the lower Darcy parameter, $D a \leq 10^{-4}$, isentropic lines are distributed around the heater of an inner circular cylinder with fewer lines near the cold outer wall. Furthermore, an increment in $D a$ augments the isentropic lines around the top area of an annulus, and it seems that the distributions of the isentropic lines are following the strength of the isothermal and streamlines contours.
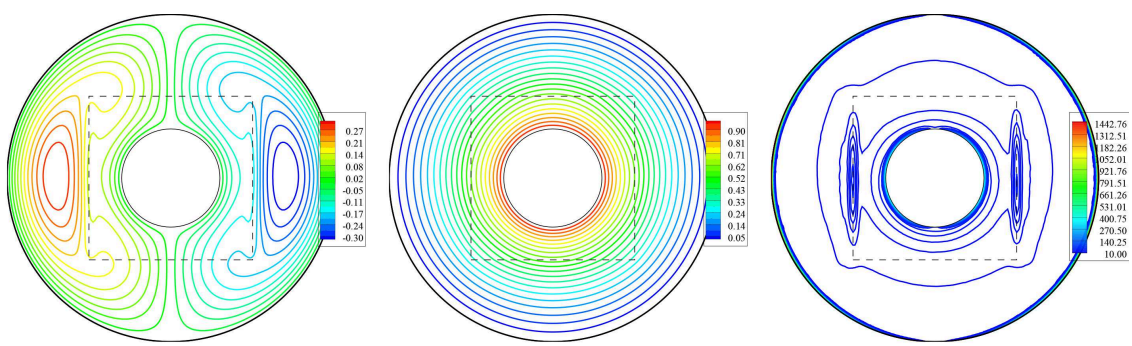

(a) $D a=10^{-5}$
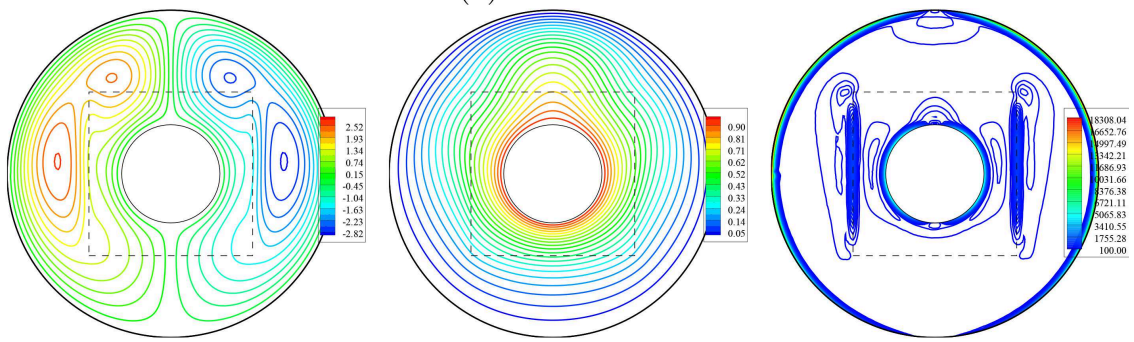

(b) $D a=10^{-4}$
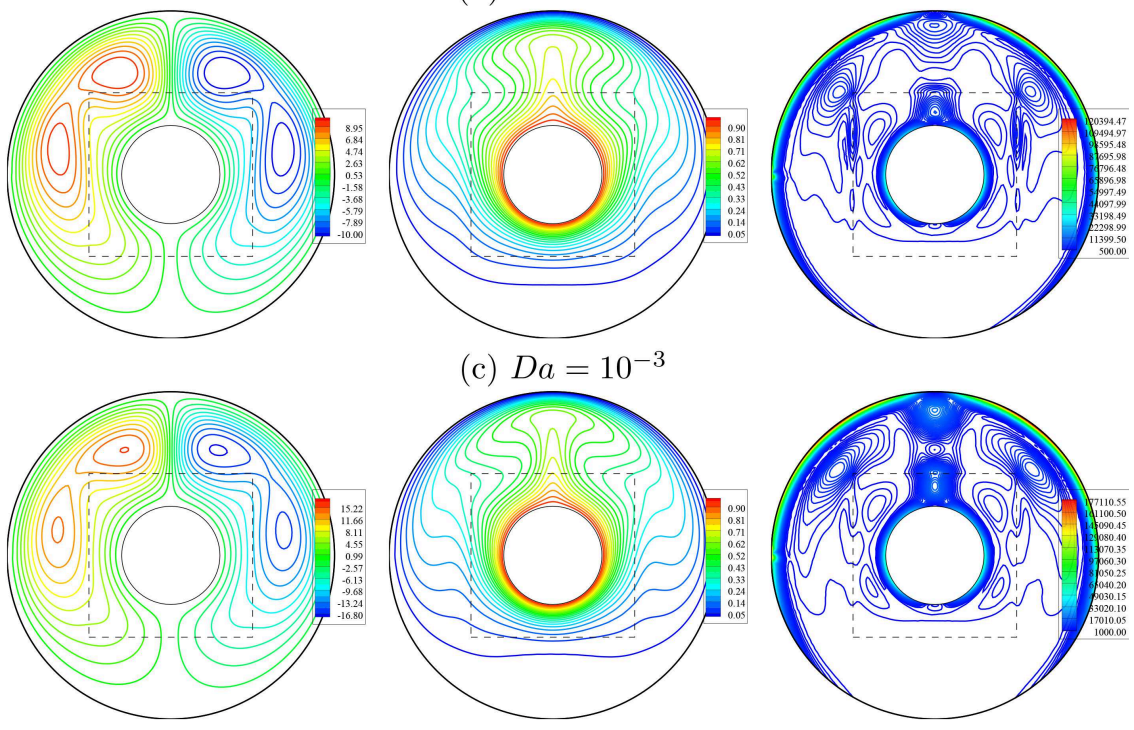

(c) $D a=10^{-3}$
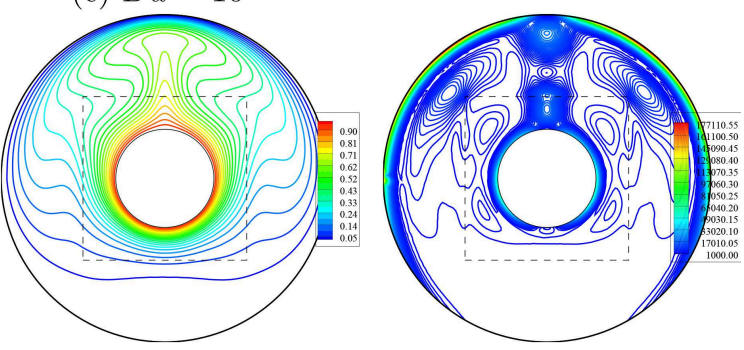

(d) $D a=10^{-2}$

Figure 5. Streamlines (left), isotherms (middle), and isentropic lines (right) with various Darcy numbers $(D a) ; \phi=0.02, \varepsilon=0.5$ and $D=0.5$.

Figure 6 presents the influences of $D a$ toward the local velocity, local temperature, and local interface Nusselt number on the interface wall within nanofluid-porous layers, $W$ at $\phi=0.02$ and $\varepsilon=0.5$. In Figure $6 \mathrm{a}$, it is observed that there are three peaks on the local velocity $V$ across $W$ around the locations of $W=0.5,1$, and 1.5 , in which the local velocity is $(V \leq 0)$. Further, the local velocity, $V$ is improving clearly as $D a$ boosts. Figure $6 \mathrm{~b}$ shows fluctuations in the local temperature across $W$ below the variations on $D a$. When $(0.5 \leq W \leq 1)$, the value of $D a=10^{-4}$ provides the highest values of the local temperature and in the other parts of $W$, the highest values of the local temperature are obtained at $D a=10^{-5}$ and the lowest at $D a \geq 10^{-3}$. The results are returning to the distributions 
of the isothermal lines through an annulus, as reported above in Figure 5. In Figure 6c, the local interface Nusselt number across the interface wall $W$ showed fluctuations under the effects of $D a$.

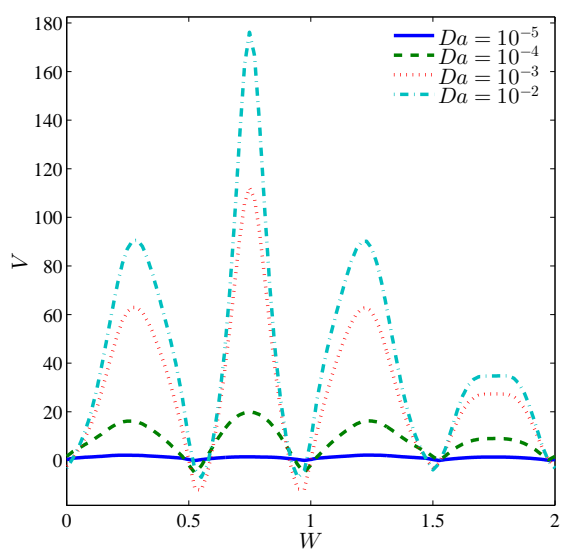

(a)

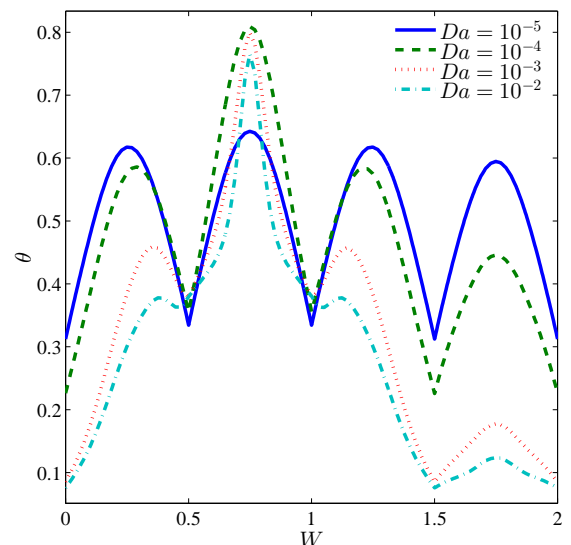

(b)

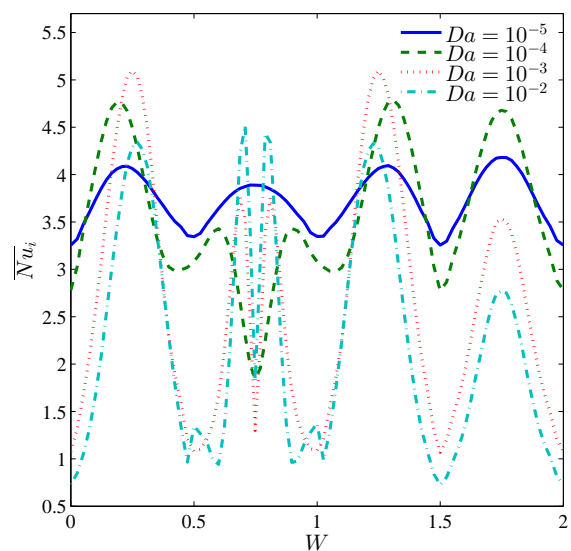

(c)

Figure 6. Local velocity (a), local temperature (b) and local interface Nusselt number (c) at the interface wall between nanofluid-porous layers for different $D a ; \phi=0.02, \varepsilon=0.5$ and $D=0.5$.

Figure 7 depicts the impacts of porosity parameter $\varepsilon$, at the streamlines, isotherms, and isentropic lines at $D a=10^{-3}, \phi=0.02$ and $D=0.5$. It is observed that the value of $|\psi|_{\max }$ increases by $32.37 \%$, as $\varepsilon$ raises from 0.2 to 0.8 . Thus, the streamline contours are affected by the variations on $\varepsilon$. There are minor reductions in the isothermal lines according to an increase in the value of $\varepsilon$. Whilst, the contours of the isentropic lines are clearly improved within an annulus according to an increase in the value of $\varepsilon$. Figure 8 presents the influences of $\varepsilon$ on the local velocity, local temperature, and local interface Nusselt number on the interface surface within nanofluid-porous layers, $W$ at $D a=10^{-3}$, $\phi=0.02$ and $D=0.5$. The local velocity, $V$, showed a clear increase from $W=0.5$ to 1 , as $\varepsilon$ and in the other parts of the interface wall, there is almost no change on the values of $V$ according to the variations on $\varepsilon$. Further, there is a slight enhancement in the local temperature and local interface Nusselt number according to a reduction in $\varepsilon$. The physical representations of these results are returning to the medium's porosity definition as it is characterized from the ratio of pore volume and the total volume of material, and an increase in $\varepsilon$ towards the unity represents the free fluid. 

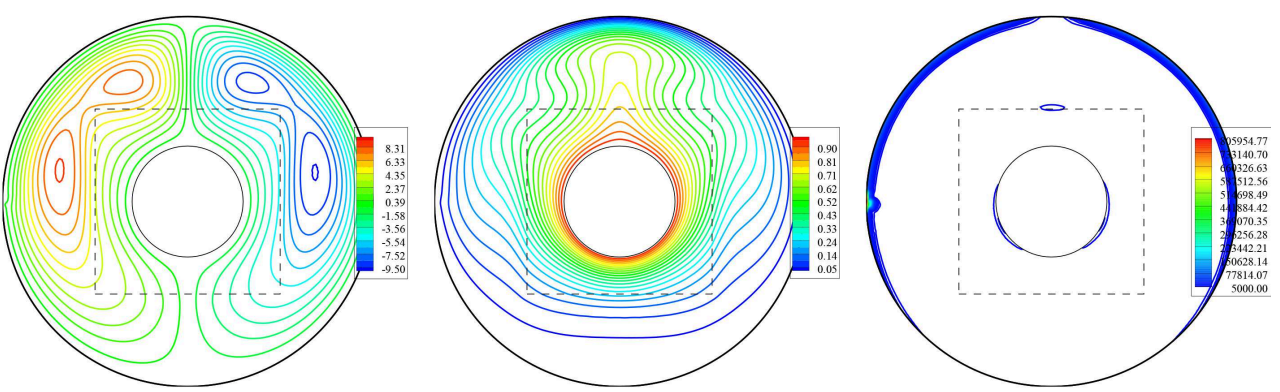

(a) $\varepsilon=0.2$
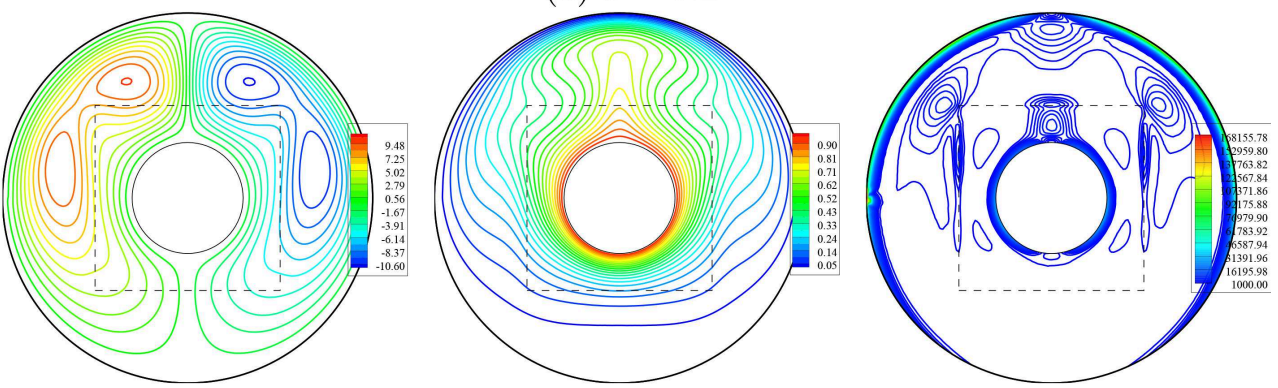

(b) $\varepsilon=0.4$
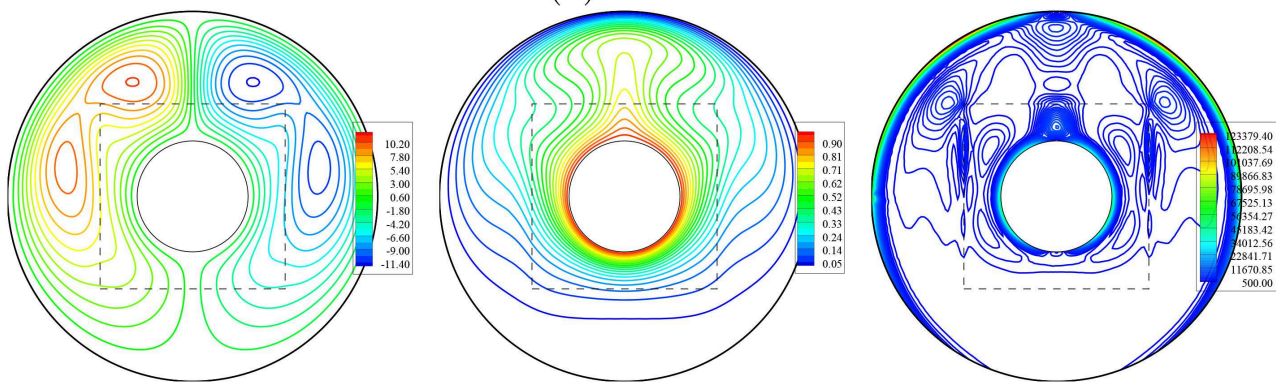

(c) $\varepsilon=06$
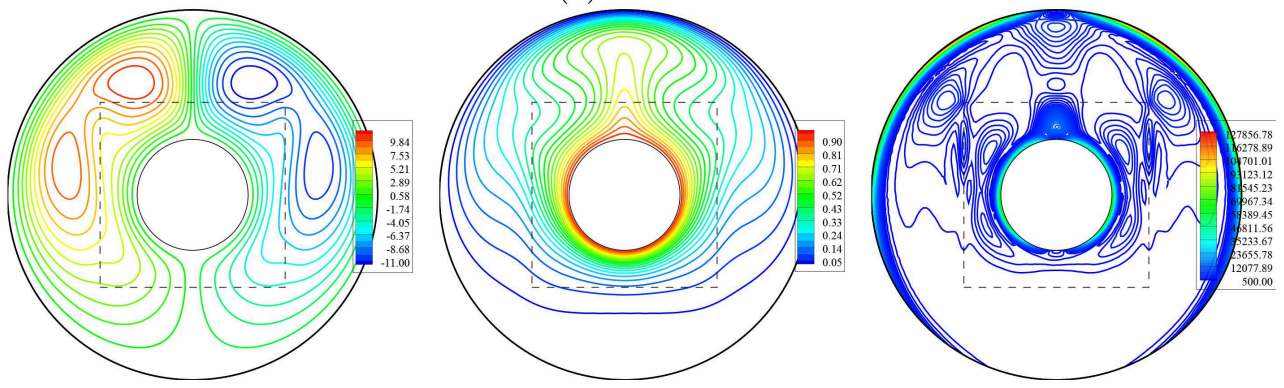

(d) $\varepsilon=0.8$

Figure 7. Streamlines (left), isotherms (middle), and isentropic lines (right) with various porosity of the medium $(\varepsilon) ; D a=10^{-3}, \phi=0.02$ and $D=0.5$. 


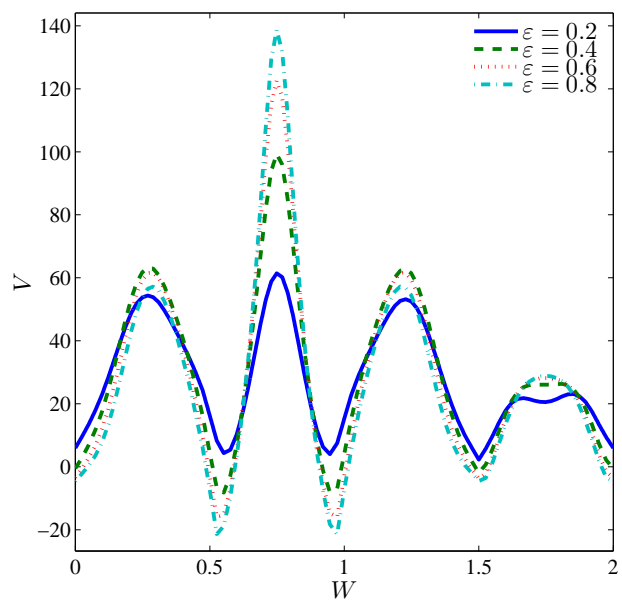

(a)

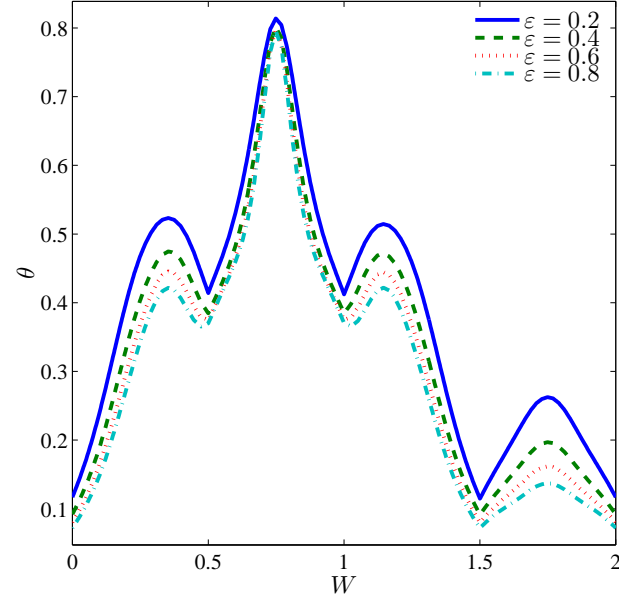

(b)

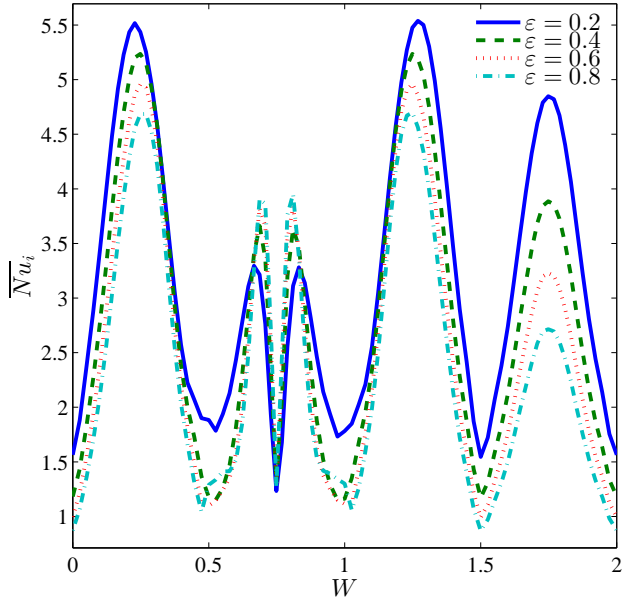

(c)

Figure 8. Local velocity (a), local temperature (b) and local interface Nusselt number (c) at the interface wall between nanofluid-porous layers for different $\varepsilon ; D a=10^{-3}, \phi=0.02$ and $D=0.5$.

The dependency of the streamlines, isotherms, and isentropic lines on the various dimensionless length of porous layer $D$ at $D a=10^{-3}, \phi=0.02$ and $\varepsilon=0.5$ have been shown in Figure 9. The first remark is that $|\psi|_{\max }$ reduces by $25 \%$ as $D$ raises from 0.4 to 0.7. The reason returns to the nanofluid flow's porous resistance within an annulus at an extra length of a porous layer $D$. There is a slight enhancement in the isothermal contours according to an increase in $D$. Thus, the contours of isentropic lines are influenced by the variations on the length of a porous layer $D$. Figure 10 presents the local velocity, local temperature, and local interface Nusselt number at the interface wall between nanofluidporous layers under the variations on $D$ at $D a=10^{-3}, \phi=0.02$ and $\varepsilon=0.5$. It is observed that the lower value of $D$ gives the highest values of the local velocity at the interface wall. Due to the expansion of $D$, the values of local velocity fluctuate between positive and negative values, as shown previously for an interface wall's location over the streamlines (Figure 9). Further, the local temperature's highest peak across the interface wall appears at the lowest value of $D=0.4$. The profiles of $\overline{N u}_{i}$ are decreasing according to an increment in $D$. 

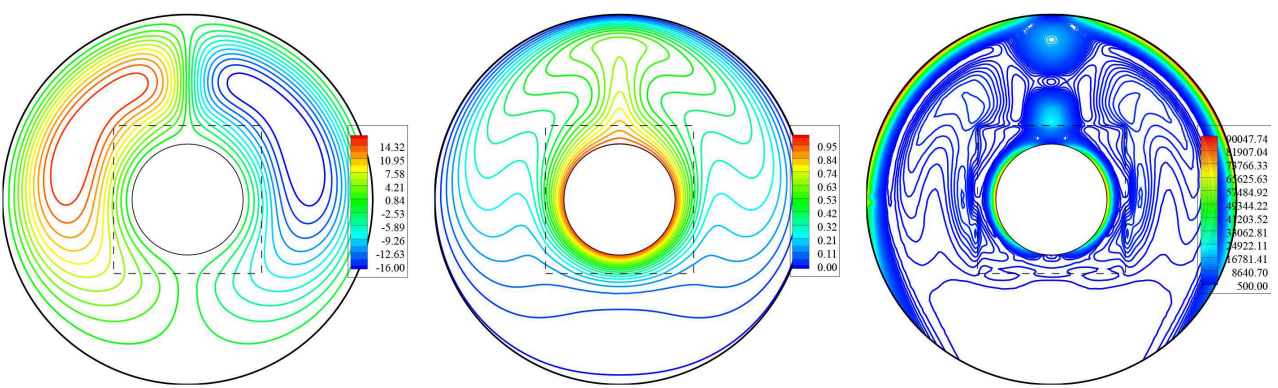

(a) $D=0.4$
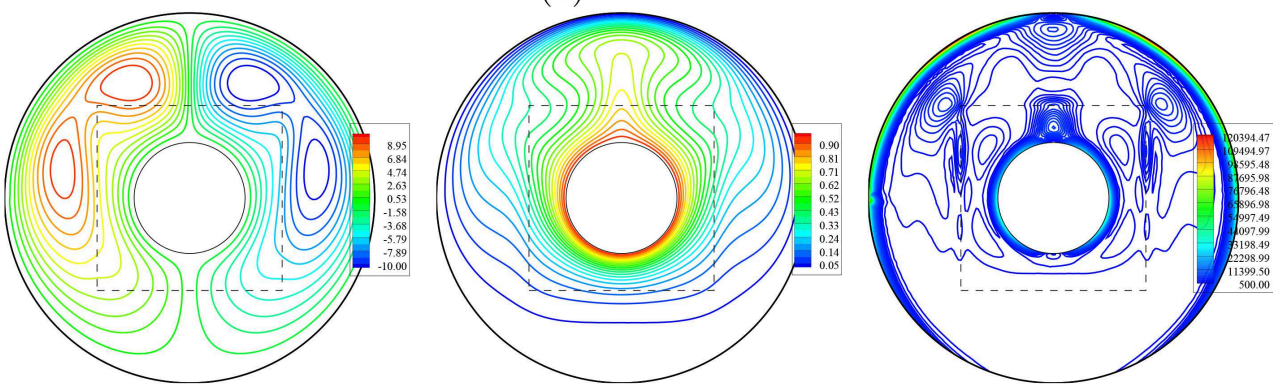

(b) $D=0.5$
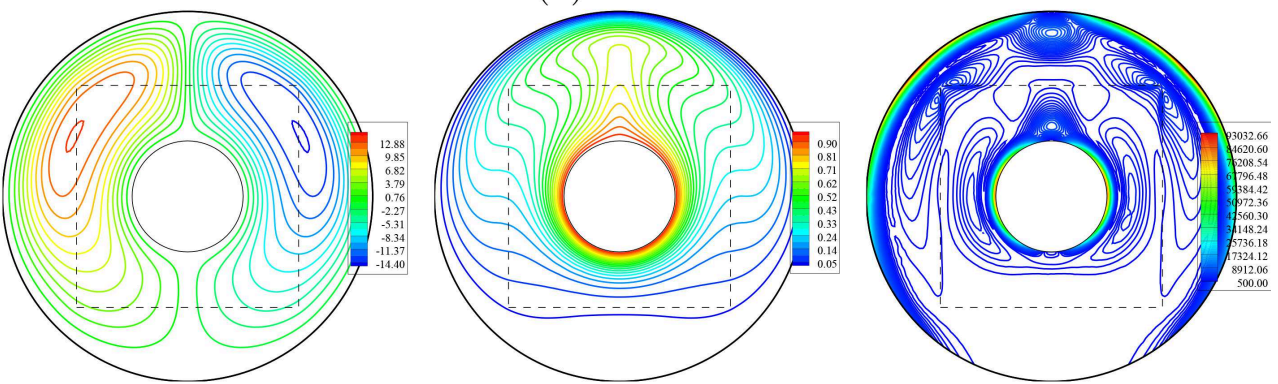

(c) $D=0.6$
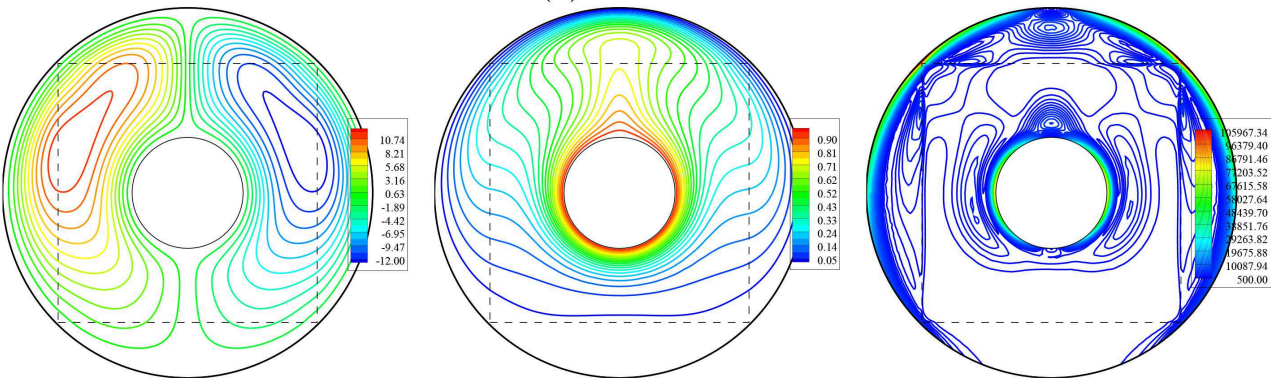

(d) $D=0.7$

Figure 9. Streamlines (left), isotherms (middle), and isentropic lines (right) with various dimensionless length of porous layer $(D) ; D a=10^{-3}, \phi=0.02$ and $\varepsilon=0.5$.

Figure 11 introduces the changes of average Nusselt number $\left(\overline{N u}_{n f}\right)$, average temperature $\left(\theta_{\text {avg }}\right)$, and Bejan number $(B e)$ during various $D a$ and $\phi$ at $\varepsilon=0.5$ and $D=0.5$. It is remarked that $\overline{N u}_{n f}$ is slightly enhanced as $\phi$ increases and $\overline{N u}_{n f}$ is strongly improved as $D a$ powers. There are minor changes on the $\theta_{\text {avg }}$ under the variations on $\phi$. A reduction in $D a$ augments the value of $\theta_{\text {avg. }}$. Further, the value of $B e$ showed a similar tendency as $\theta_{\text {avg }}$ under the influences of $\phi$ and $D a$. The values of $B e$ increase as $D a$ decreases, and there is a minor enhancement in $B e$ under the augmentation in $\phi$. The outcomes show improved heat transfer through high porous resistance (lower $D a$ ). 


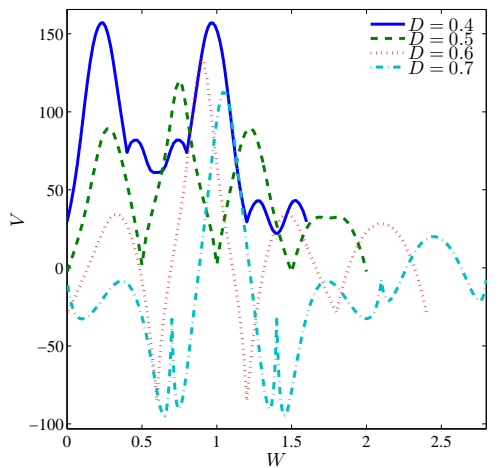

(a)

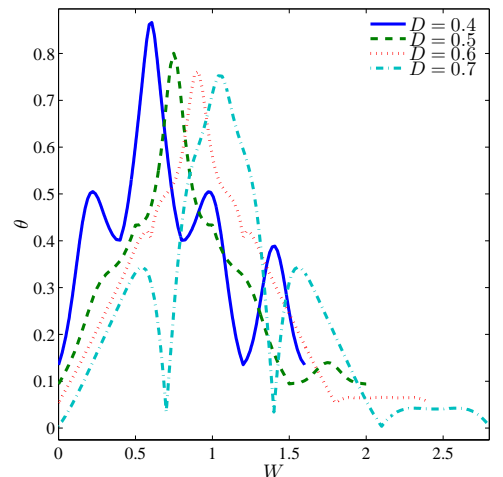

(b)

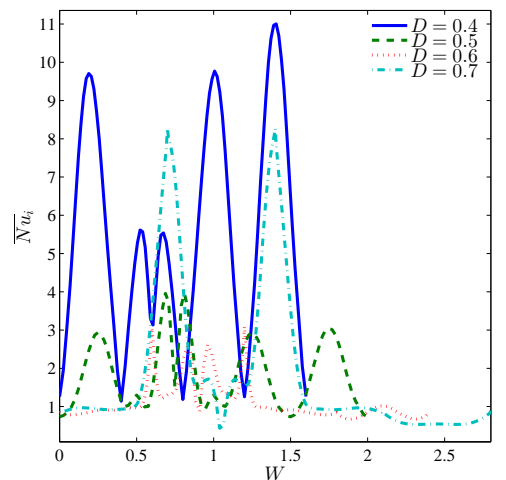

(c)

Figure 10. Local velocity (a), local temperature (b) and local interface Nusselt number (c) at the interface wall between nanofluid-porous layers for different $D ; D a=10^{-3}, \phi=0.02$ and $\varepsilon=0.5$.

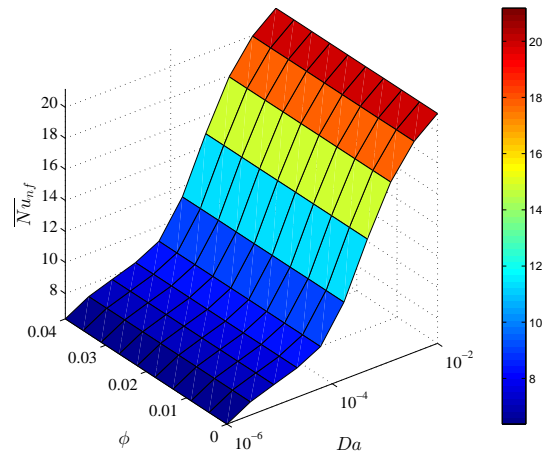

(a)

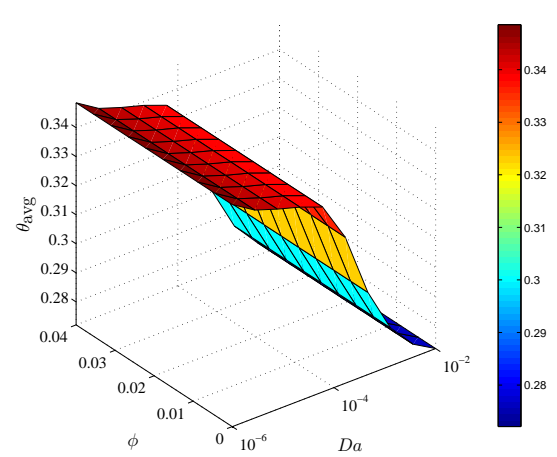

(b)

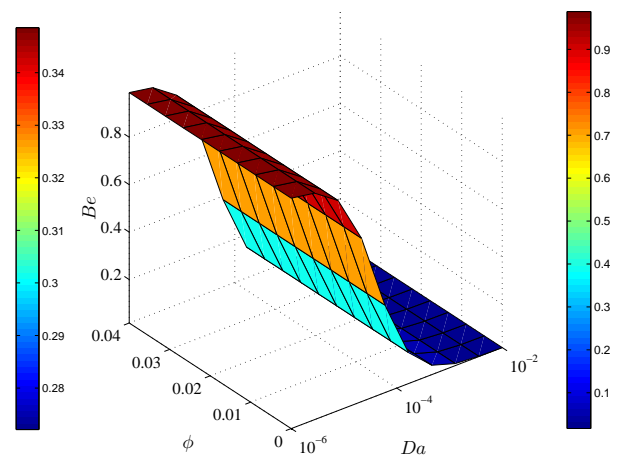

(c)

Figure 11. Variations of (a) average Nusselt number, (b) average temperature and (c) Bejan number for different $D a$ and $\phi$ at $\varepsilon=0.5$ and $D=0.5$. 
Figure 12 indicates the dependency of $\overline{N u}_{n f}, \theta_{\mathrm{avg}}$, and Bejan number $B e$ on $D a$ and $\varepsilon$ at $\phi=0.02$ and $D=0.5$. In this figure, an augmentation in $D a$ raises $\overline{N u}_{n f}$ and declines the values of $\theta_{\text {avg }}$ and $B e$. The physical reason returns to the high porous resistance of the nanofluid movements at a lower Darcy parameter. A decrease in $D a$ enhances the heat transfer within a circular cylinder. Further, there are minor changes in $\overline{N u}_{n f}, \theta_{\text {avg }}$ and $B e$ under the variations of $\varepsilon$. It is determined that the impacts of $D a$ on $\overline{N u}_{n f}, \theta_{\text {avg }}$ and $B e$ are high enough, in which the impacts of $\phi$ and $\varepsilon$ can be neglected.

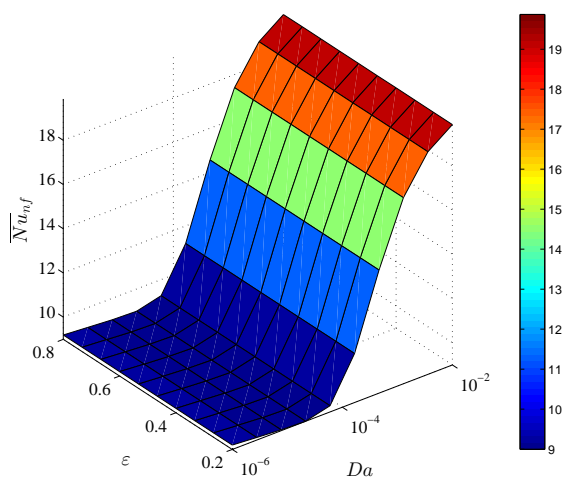

(a)

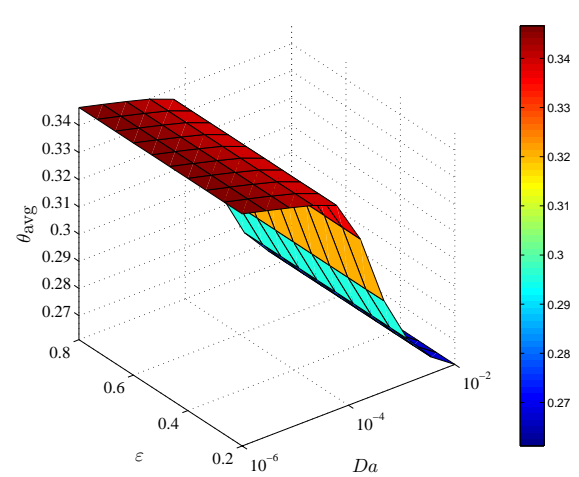

(b)

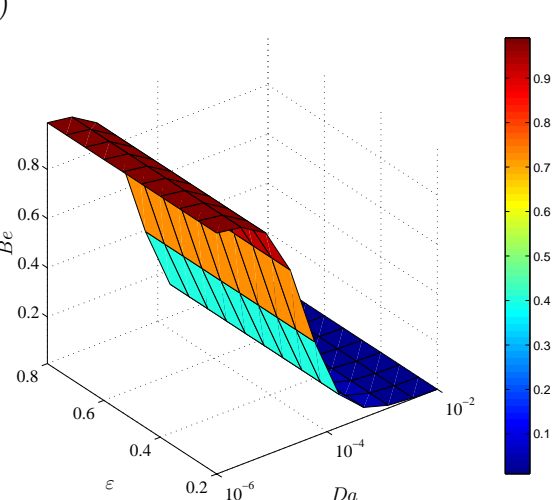

(c)

Figure 12. Variations of (a) average Nusselt number, (b) average temperature and (c) Bejan number for different $D a$ and $\varepsilon$ at $\phi=0.02$ and $D=0.5$.

Figure 13 shows the influences of $\phi$ and $\varepsilon$ on $\overline{N u}_{n f}, \theta_{\text {avg }}$ and $B e$ at $D a=10^{-3}$ and $D=0.5$. Here, an increment in $\phi$ improves the values of $\overline{N u}_{n f}$ and declines the values of $\theta_{\text {avg }}$ and $B e$. Furthermore, there are almost no changes in $\overline{N u}_{n f}, \theta_{\text {avg }}$ and Be below the variations of $\varepsilon$. The physical reason returns to low porous resistance according to the alterations on $\varepsilon$. In Figure 14, for any value of $D$, an increment in $D a$ is still enhancing $\overline{N u}_{n f}$ and reduces $\theta_{\text {avg }}$ and $B e$. Further, the values of $\overline{N u}_{n f}, \theta_{\text {avg }}$ and $B e$ have little changes according to the variation on the dimensionless length of porous layer $D$ at different values of $D a$. 


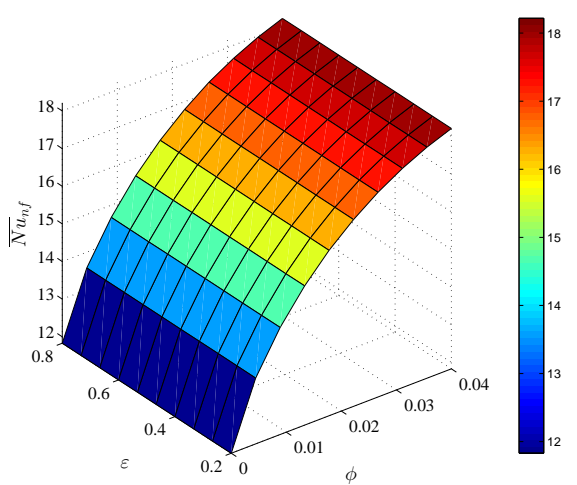

(a)

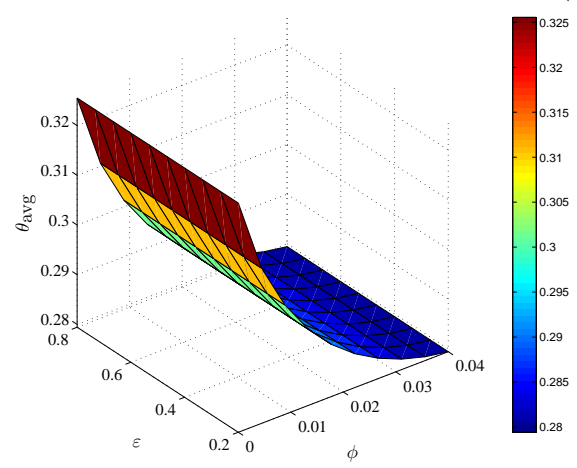

(b)

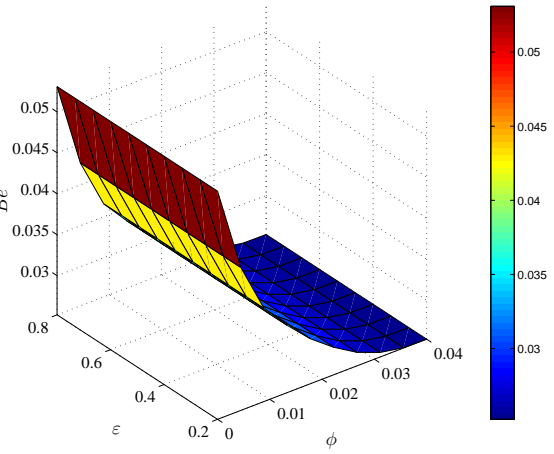

(c)

Figure 13. Variations of (a) average Nusselt number, (b) average temperature and (c) Bejan number for different $\phi$ and $\varepsilon$ at $D a=10^{-3}$ and $D=0.5$.

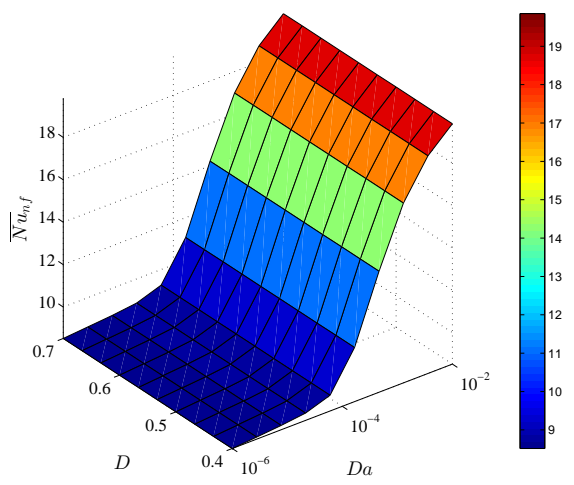

(a)

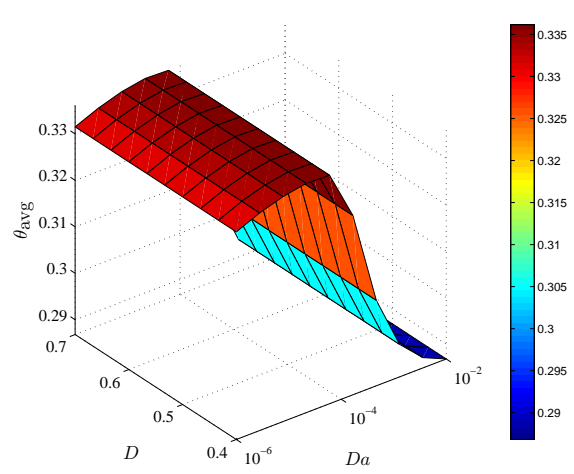

(b)

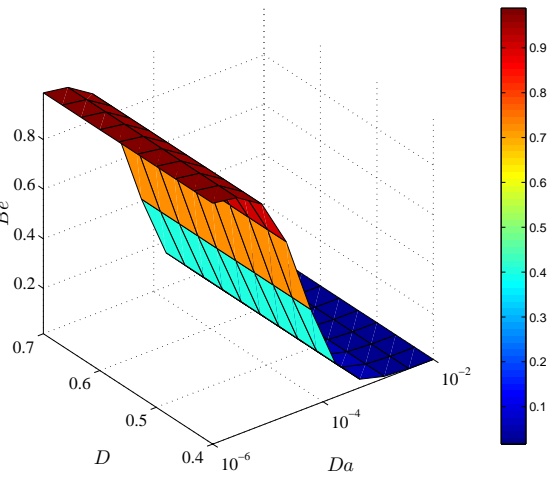

(c)

Figure 14. Variations of (a) average Nusselt number, (b) average temperature and (c) Bejan number for different $D a$ and $D$ at $\phi=0.02$ and $\varepsilon=0.5$. 


\section{Conclusions}

This study applied the entropy generation on nanofluid superposed horizontal annulus porous layers via the fluid flow and heat transfer characteristics. Here, the values of local velocity, local temperatures, local Nusselt numbers, average Nusselt number, average temperature, and Bejan number are measured in the interface surface within nanofluid-porous layers. The following points are summarizing the main findings:

- Increasing the Darcy parameter from $10^{-5}$ to $10^{-2}$ powers the value of streamlines' maximum and strengthens the intensity of the isotherms and isentropic lines within an annulus due to higher porous resistance at a lower Darcy parameter.

- An augmentation in the Darcy parameter boosts the local velocity and average Nusselt number and reduces the average temperature and Bejan number.

- Increasing the porosity parameter augments the streamlines' maximum by $32.37 \%$ and improves the contours of isentropic lines within an annulus.

- The value of streamlines' maximum reduces by $25 \%$ as the length of a porous layer raises from 0.4 to 0.7 .

- The highest values of the local velocity and local temperature correspond with lower lengths of a porous layer.

- The profiles of the local Nusselt number decrease according to an increase in the length of a porous layer.

- Increasing the solid volume fraction improves the values of the average Nusselt number and reduces the average temperature and Bejan number.

Author Contributions: Formal analysis, A.I.A.; Funding acquisition, Z.A.S.R.; Investigation, A.M.A.; Methodology, A.I.A.; Resources, Z.A.S.R. and I.H.; Software, A.I.A.; Supervision, I.H.; Validation, A.M.A.; Writing—original draft, A.M.A.; Writing—review and editing, Z.A.S.R. All authors have read and agreed to the published version of the manuscript.

Funding: The authors would like to extend their appreciation to the Deanship of Scientific Research at King Khalid University, Abha, Saudi Arabia, for funding this work through the Research Group Project under grant number R.G.P2/144/42. Furthermore, we are grateful for the financial support received from the Universiti Kebangsaan Malaysia (UKM) research grant GP-2020-K006388.

Institutional Review Board Statement: Not applicable.

Informed Consent Statement: Not applicable.

Acknowledgments: We thank the respected reviewers for their constructive comments, which clearly enhanced the quality of the manuscript.

Conflicts of Interest: The authors declare no conflict of interest.

\section{Nomenclature}

Be Bejan number

$C_{p} \quad$ specific heat capacity $(\mathrm{J} / \mathrm{kg} \mathrm{K})$

Da Darcy number

$g \quad$ gravitational acceleration, $\left(\mathrm{m} / \mathrm{s}^{2}\right)$

GEG dimensionless global entropy generation

$D \quad$ dimensionless length of the porous layer, $(D=d / r r)$

$k$ thermal conductivity, $(\mathrm{W} / \mathrm{m} \mathrm{K})$

$K \quad$ permeability of porous medium, $\left(\mathrm{m}^{2}\right)$

$N \quad$ Avogadro number

$N_{m} \quad$ irreversibility distribution ratio related to the porous layer

$N_{n f} \quad$ irreversibility distribution ratio related to the nanofluid layer 


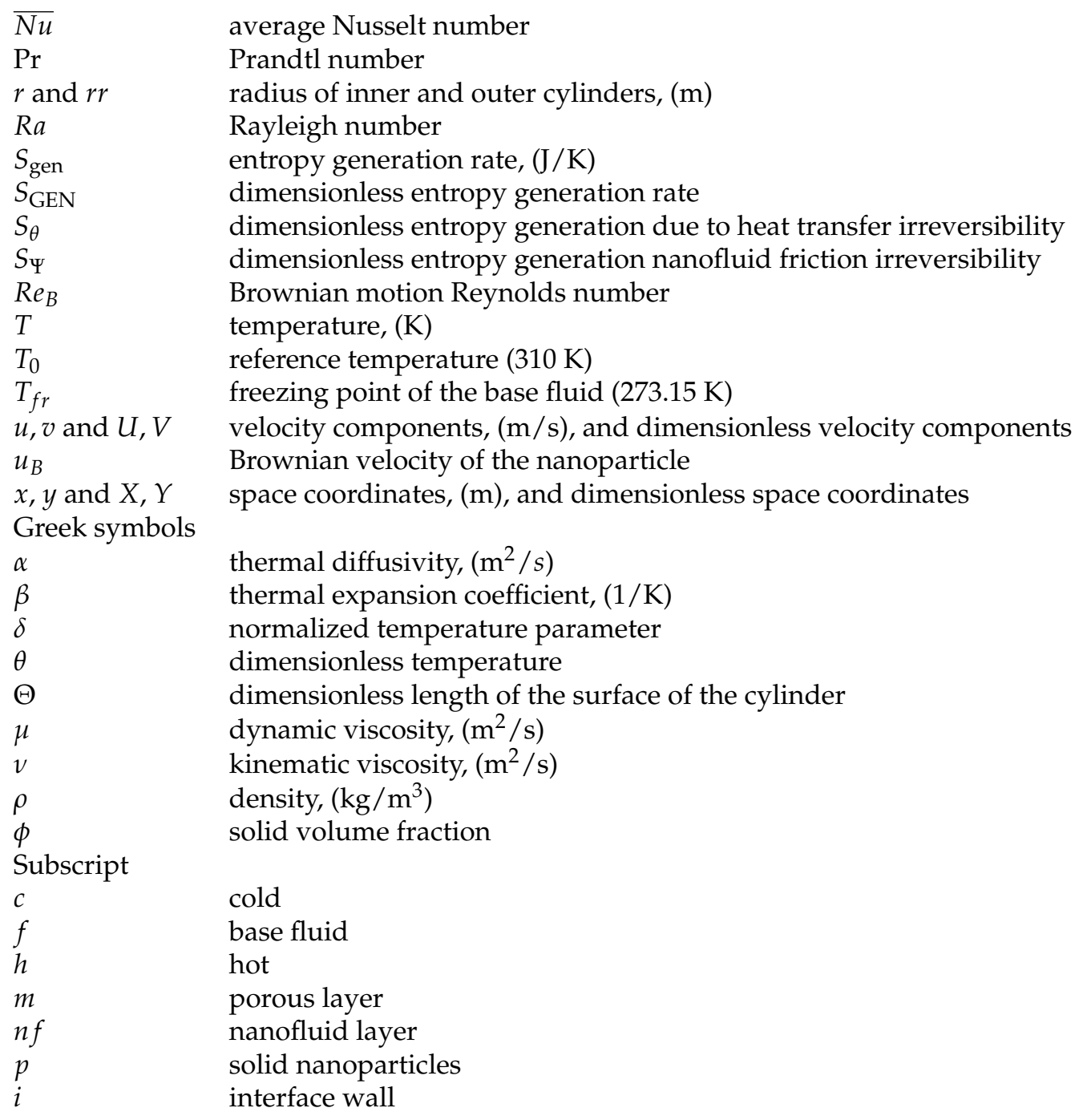

\section{References}

1. Culham, J.R.; Teertstra, P.; Yovanovich, M.M. Natural convection modeling of heat sinks using web-based tools. Electron. Cool. 2000, 6, 44-51.

2. Buchberg, H.; Catton, I.; Edwards, D.K. Natural convection in enclosed spaces-a review of application to solar energy collection. J. Heat Transf. 1976, 98, 182-188. [CrossRef]

3. Chen, H.T.; Chou, J.C. Investigation of natural-convection heat transfer coefficient on a vertical square fin of finned-tube heat exchangers. Int. J. Heat Mass Transf. 2006, 49, 3034-3044. [CrossRef]

4. Mistry, H.; Dey, S.; Bishnoi, P.; Castillo, J.L. Modeling of transient natural convection heat transfer in electric ovens. Appl. Therm. Eng. 2006, 26, 2448-2456. [CrossRef]

5. Dayem, A.M.A. Experimental and numerical performance of a multi-effect condensation-evaporation solar water distillation system. Energy 2006, 31, 2710-2727.

6. Kalaiselvam, S.; Veerappan, M.; Aaron, A.A.; Iniyan, S. Experimental and analytical investigation of solidification and melting characteristics of PCMs inside cylindrical encapsulation. Int. J. Therm. Sci. 2008, 47, 858-874. [CrossRef]

7. Wang, S.; Faghri, A.; Bergman, T.L. A comprehensive numerical model for melting with natural convection. Int. J. Heat Mass Transf. 2010, 53, 1986-2000. [CrossRef]

8. Sparrow, E.M.; Ramsey, J.W.; Kemink, R.G. Freezing controlled by natural convection. J. Heat Transfer. 1979. 101, 578-584. [CrossRef]

9. Sparrow, E.M.; Larson, E.D.; Ramsey, J.W. Freezing on a finned tube for either conduction-controlled or natural-convectioncontrolled heat transfer. Int. J. Heat Mass Transf. 1981, 24, 273-284. [CrossRef]

10. Nield, D.A.; Bejan, A. Convection in Porous Media; Springer: Berlin, Germany, 2006; Volume 3.

11. Toosi, M.H.; Siavashi, M. Two-phase mixture numerical simulation of natural convection of nanofluid flow in a cavity partially filled with porous media to enhance heat transfer. J. Mol. Liq. 2017, 238, 553-569. [CrossRef] 
12. Raizah, Z.A.S.; Aly, A.M.; Ahmed, S.E. Natural convection flow of a power-law non-Newtonian nanofluid in inclined open shallow cavities filled with porous media. Int. J. Mech. Sci. 2018, 140, 376-393. [CrossRef]

13. Alsabery, A.I.; Ismael, M.A.; Chamkha, A.J.; Hashim, I. Effect of nonhomogeneous nanofluid model on transient natural convection in a non-Darcy porous cavity containing an inner solid body. Int. Commun. Heat Mass Transf. 2020, $110,104442$. [CrossRef]

14. Miroshnichenko, I.V.; Sheremet, M.A.; Oztop, H.F.; Abu-Hamdeh, N. Natural convection of alumina-water nanofluid in an open cavity having multiple porous layers. Int. J. Heat Mass Transf. 2018, 125, 648-657. [CrossRef]

15. Cho, C.C. Effects of porous medium and wavy surface on heat transfer and entropy generation of Cu-water nanofluid natural convection in square cavity containing partially-heated surface. Int. Commun. Heat Mass Transf. 2020, 119, 104925. [CrossRef]

16. Selimefendigil, F.; Öztop, H.F. Magnetohydrodynamics forced convection of nanofluid in multi-layered U-shaped vented cavity with a porous region considering wall corrugation effects. Int. Commun. Heat Mass Transf. 2020, 113, 104551. [CrossRef]

17. Mehryan, S.A.M.; Ghalambaz, M.; Chamkha, A.J.; Izadi, M. Numerical study on natural convection of Ag-MgO hybrid/water nanofluid inside a porous enclosure: A local thermal non-equilibrium model. Powder Technol. 2020, 367, 443-455. [CrossRef]

18. Alsabery, A.I.; Ismael, M.A.; Chamkha, A.J.; Hashim, I.; Abulkhair, H. Unsteady flow and entropy analysis of nanofluids inside cubic porous container holding inserted body and wavy bottom wall. Int. J. Mech. Sci. 2021, 193, 106161. [CrossRef]

19. Raizah, Z.A.S.; Aly, A.M.; Ahmed, S.E. Natural convection flow of a nanofluid-filled V-shaped cavity saturated with a heterogeneous porous medium: Incompressible smoothed particle hydrodynamics analysis. Ain Shams Eng. J. 2021, 12, $2033-2046$. [CrossRef]

20. Aly, A.M.; Raizah, Z.A.S. Incompressible smoothed particle hydrodynamics simulation of natural convection in a nanofluid-filled complex wavy porous cavity with inner solid particles. Phys. A Stat. Mech. Its Appl. 2020, 537, 122623. [CrossRef]

21. Javaherdeh, K.; Karimi, H.; Khojasteh, A. Numerical study of heat transfer enhancement of non-Newtonian nanofluid in porous blocks in a channel partially. Powder Technol. 2021, 383, 270-279. [CrossRef]

22. Rao, P.S.; Barman, P. Natural convection in a wavy porous cavity subjected to a partial heat source. Int. Commun. Heat Mass Transf. 2021, 120, 105007. [CrossRef]

23. Wang, L.; Huang, C.; Hu, J.; Shi, B.; Chai, Z. Effects of temperature-dependent viscosity on natural convection in a porous cavity with a circular cylinder under local thermal non-equilibrium condition. Int. J. Therm. Sci. 2021, 159, 106570. [CrossRef]

24. Esfe, M.H.; Barzegarian, R.; Bahiraei, M. A 3D numerical study on natural convection flow of nanofluid inside a cubical cavity equipped with porous fins using two-phase mixture model. Adv. Powder Technol. 2020, 31, 2480-2492. [CrossRef]

25. Nithiarasu, P.; Seetharamu, K.N.; Sundararajan, T. Natural convective heat transfer in a fluid saturated variable porosity medium. Int. J. Heat Mass Transf. 1997, 40, 3955-3967. [CrossRef]

26. Corcione, M. Empirical correlating equations for predicting the effective thermal conductivity and dynamic viscosity of nanofluids. Energy Convers. Manag. 2011, 52, 789-793. [CrossRef]

27. Ilis, G.G.; Mobedi, M.; Sunden, B. Effect of aspect ratio on entropy generation in a rectangular cavity with differentially heated vertical walls. Int. Commun. Heat Mass Transf. 2008, 35, 696-703. [CrossRef]

28. Alsabery, A.; Ismael, M.; Chamkha, A.; Hashim, I. Numerical investigation of mixed convection and entropy generation in a wavy-walled cavity filled with nanofluid and involving a rotating cylinder. Entropy 2018, 20, 664. [CrossRef]

29. Alsabery, A.I.; Hashim, I.; Hajjar, A.; Ghalambaz, M.; Nadeem, S.; Saffari Pour, M. Entropy generation and natural convection flow of hybrid nanofluids in a partially divided wavy cavity including solid blocks. Energies 2020, 13, 2942. [CrossRef]

30. Bejan, A. Second-law analysis in heat transfer and thermal design. Adv. Heat Transf. 1982, 15, 1-58.

31. Kuehn, T.H.; Goldstein, R.J. An experimental and theoretical study of natural convection in the annulus between horizontal concentric cylinders. J. Fluid Mech. 1976, 74, 695-719. [CrossRef]

32. Beckermann, C.; Viskanta, R.; Ramadhyani, S. Natural convection in vertical enclosures containing simultaneously fluid and porous layers. J. Fluid Mech. 1988, 186, 257-284. [CrossRef]

33. Bergman, T.L.; Incropera, F.P. Introduction to Heat Transfer, 6th ed.; Wiley: New York, NY, USA, 2011. 